\title{
Correlation of Next-Generation Sequencing Based Genotypic Profiles with MICM Characteristics in NPM1 Mutated Acute Myeloid Leukemia
}

\author{
Biao Wang \\ Changzhou First People's Hospital https://orcid.org/0000-0003-1557-5045 \\ Bin Yang \\ Changzhou First People's Hospital \\ Wei Wu \\ Changzhou First People's Hospital \\ Xuan Liu \\ Shengjing Hospital of China Medical University \\ Haiqian Li ( $\triangle$ lhz0125@163.com ) \\ Changzhou First People's Hospital https://orcid.org/0000-0002-5887-7142
}

\section{Research article}

Keywords: NPM1, FLT3-ITD, acute myeloid leukemia, immunophenotype, next-generation sequencing

Posted Date: October 27th, 2020

DOI: https://doi.org/10.21203/rs.3.rs-41514/v1

License: (c) (i) This work is licensed under a Creative Commons Attribution 4.0 International License. Read Full License

Version of Record: A version of this preprint was published at BMC Cancer on July 8th, 2021. See the published version at https://doi.org/10.1186/s12885-021-08455-7. 


\section{Abstract}

The purpose of this study was to analyze association between next-generation sequencing (NGS) genomic profile and conventional MICM characteristics in patients with acute myeloid leukemia (AML) with NPM1 mutation (NPM1 ${ }^{\text {mut }}$ ). We selected $238 N P M 7^{\text {mut }}$ patients with available NGS information on 112 genes related to blood diseases, using $X 2$ and MannWhitney $\mathrm{U}$ test to analyze the distribution correlation between genomic alterations and MICM parameters. Compared with NPM1 mut $_{\text {/ FLT3-TTD }}^{(-)}$, the NPM1 mut $_{\text {FLT3-TTD }}^{(+)}$group presented a slightly common M5 [78/143 (54.5\%) vs. 64/95 (67.4\%); $P=0.048]$, more higher CD34 and CD7 expression rates (CD34: 20.6\% vs. 47.9\%, $P<0.001 ; \mathrm{CD} 7: 29.9 \%$ vs. $61.5 \%, P<0.001$ ), and lack of favorable- and adverse-risk karyotypes (6.4\% vs. $0 \% ; P=0.031)$. In entire NPM1 ${ }^{\text {mut }}$ cohort, totaling 240 NPM1 mutation events were identified, of whom 10 (10/240, 4.2\%) were missense types. When confining analysis to the 205 cases with $N P M 7^{\text {mut }}$ insertions/deletions type and normal karyotype, multivariable logistic analysis showed that FLT3-TDD was positively correlated with CD34 and CD7 expression ( $H R=5.29$ [95\% Cl 2.64-10.60], $P<0.001 ; H R=3.47$ [95\% Cl 1.79-6.73], $P<0.001$; respectively). Ras-pathway mutation was positively correlated with HLA-DR expression ( $H R=4.05$ [95\% $\mathrm{Cl} 1.70-9.63], P=0.002)$, and KRAS mutation negatively with MPO expression ( $H R=0.18$ [95\% $\mathrm{Cl} 0.05-0.62$ ], $P=0.007)$. DNMT3A-R882 was positively correlated with CD7 and HLA-DR expression $(H R=3.59$ [95\% $\mathrm{Cl} 1.80-7.16$ ], $P<0.001 ; H R=13.41$ [95\% Cl 4.56-39.45], $P<0.001$; respectively). DNMT3A mutation was negatively correlated with MPO expression ( $H R=0.35$ [95\% $\mathrm{Cl} 1.48-8.38], P=0.004)$. TET2/ IDH1 mutations were negatively correlated with CD34 and CD7 expression $(H R=0.26$ [95\% Cl 0.11-0.62], $P=0.002$; $H R=0.30$ [95\% Cl 0.14-0.62], $P=0.001$; respectively), and positively with MPO expression ( $H R=3.52$ [95\% $\mathrm{Cl} 1.48-8.38], P=0.004)$. $N P M 7^{\text {mut }}$ co-existing mutations in signaling pathways (FLT3-TTD and Ras-pathway) and methylation modifiers (DNMT3A and TET2/ IDH1) are linked with the expression of CD34, CD7, HLA-DR and MPO, thereby providing a mechanism explanation for the immunophenotypic heterogeneity of this AML entity.

\section{Background}

The human NPM1 gene, located on chromosome 5q35.1 and containing 12 exons, encodes for a nucleolar phosphoprotein which possesses multifunctions including chromatin remodeling, ribosome biogenesis, genomic stability, regulation of tumor suppressors and transcription factors.(1-3) Given its biological diversity and important role, the functional category of NPM1 belongs to a separate one according to TCGA data.(4)

NPM1 gene abnormalities are involved in fusion(5), deletion(2) and mutation, therein, the mutation is most largely studied. The incidence of NPM1 mutation (NPM1 ${ }^{\text {mut }}$ ) occupies approximately one-third of cases with de novo acute myeloid leukemia (AML), and up to 60\% in normal karyotype (NK) AML. $(6,7)$ The clinicobiological presentation of $N P M 7^{\text {mut }}$ AML are characterized by multiple aspects of MICM typing. For instance, French-American-British (FAB) morphologies commonly have monocytic differentiation (M4 or M5) $(8,9)$, and are likely to have cup-like nuclei.(10) Immunophenotypically, most $N P M 7^{\text {mut }}$ cases showed CD34 negativity.(11) According to analysis of the myeloid blast population, nearly half of NPM7 ${ }^{\text {mut }}$ patients

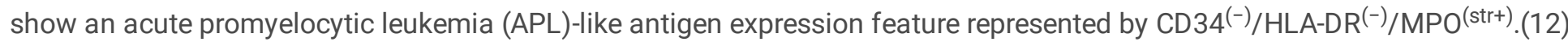
$N P M 1^{\text {mut }} A M L$ mainly arises in a NK situation, mutually exclusive with recurrent cytogenetic abnormalities. $(7,13) N P M 1^{\text {mut }}$ AML has unique gene expression profiles, especially the overexpression of HOX family members.(14)

Immunophenotype is not only used in the differential diagnosis of $\mathrm{AML}$, but also has prognostic value. $\operatorname{The} \operatorname{CD} 34^{(+)}(11,15)$, leukemic stem cells (LSCs) phenotype CD34 $4^{(+)} / \mathrm{CD} 38^{(-)} / \mathrm{CD}_{123^{(+)}}(16)$, APL-like phenotype CD34(-)/HLA-DR ${ }^{(-)} / \mathrm{MPO}^{(\mathrm{str}+)}(12)$

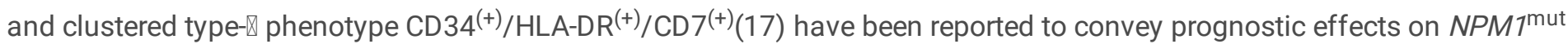
AML. However, data on genetic information were less integrated into analysis in those relatively earlier studies. In recent years, studies regarding prognostic heterogeneity in $N P M 7^{\text {mut }} A M L$ mainly focused on cytogenetic and gene mutations. Comutations in $\operatorname{DNMT3A}(18), \operatorname{TET} 2(19,20)$ or IDH1/2(21, 22) have been shown as an adverse predictor, and NRAS(23), FLT3$\operatorname{TKD}(24)$ or moCEBPA(25) as a favorable predictor on clinical outcome in NPM1 ${ }^{\text {mut }} / F L T 3$ ITD $^{(-) / l o w}$ AML. 
Because $N P M 7^{\text {mut }} A M L$ is mainly seen in an intermediate-risk cytogenetics, especially in NK background, we hypothesize that the diversity of leukemic phenotypes depends to a certain extent on the heterogeneity of coexisting gene mutations in this subtype of AML. Whole genome or exosome sequencing revealed an average of 13 mutations in $\mathrm{AML}(7)$, indicating the interplay across mutations as an important pathomechanism of leukemic development and overt onset.

In addition, the $N P M 7^{\text {mut }}$ in association with prognostication is generally described as insertions and/or deletions (indels), which is predominantly characterized by 4 base pair (bp) insertion in the C-terminus within exon 12 and results in a frameshift consequence. However, data involving other mutational types of $N P M 7^{\text {mut }}$ were scarcely reported. Moreover, the types of $N P M 7^{\text {mut }}$ were not specifically designated in AML classification and treatment guidelines. $(26,27)$ The development of largescale parallel sequencing technology, with its enlargement of higher throughput and wider coverage, is bound to detect more diversified mutational loci and types within the NPM1 gene, as well as more concurrent mutations.

In this study, we selected newly diagnosed patients with de novo NPM7 ${ }^{\text {mut }} \mathrm{AML}$ and evaluate the correlations of MICM features with next-generation sequencing (NGS) determining genetic alterations in 112 genes related to blood diseases, aiming to profoundly understand the clinicopathological heterogeneity of this AML subtype.

\section{Patients And Methods}

\section{Patient selection and MICM workup}

We performed a retrospective review of newly diagnosed de novo AML patients in our institute and Shengjing Hospital of China Medical University from October 2014 to September 2019. AML diagnosis fulfilled the World Health Organization (WHO) criteria(28), according to which the MICM workup included cytomorphology, immunotyping, chromosome karyotyping and fluorescence in situ hybridization (FISH), molecular biology and gene mutation analysis (see below). The cytomorphological subtype was based on the FAB classification. Immunophenotyping was performed on freshly EDTA-anticoagulated or heparinized bone marrow (BM) or peripheral blood (PB) samples obtained at the time of initial diagnosis. The four-color analysis was conducted on FACSCalibur (Becton-Dickinson, USA) using the following sets of FITC (fluoresceinisothiocyanate), PE (phycoerythrin), PerCP (peridinin-chlorophyll-protein) and APC (allophycocyanin) labeled mouse antihuman fluorescent monoclonal antibodies: 1) CD34/CD10/CD45/CD19; 2) CD7/CD117/CD45/CD33; 3) CD9/CD2/CD45/CD56; 4) CD15/CD38/CD45/HLA-DR; 5) CD16/CD13/CD45/CD11b; 6) CD4/CD64/CD45/CD14; 7) cMPO/cCD79a/CD45/cCD3; and 8) TdT/CD123/CD45/HLA-DR. G-band karyotyping analysis was conducted using BM aspirate samples. When obtaining BM samples was difficult, PB was used instead. A total of 20 metaphase cells were analyzed for each patient, chromosomal abnormalities described according to the International System for Human Cytogenetic Nomenclature.(29) Additionally, KMT2A (MLL) rearrangements (11q23 abnormality) were verified by fluorescence in situ hybridization (FISH) using Dual-Color, Break-Apart Rearrangement Probe (Vysis, USA), and TP53 deletions (17p-) by locus-specific Probe (Vysis, USA), respectively. The study was conducted in accordance with the Declaration of Helsinki and was approved by the institutional review board (IRB) of all the participating institutions. All patients provided written informed consent for using their records.

\section{Detection of mutations by NGS and conventional methods}

Genomic DNA extraction (Qiagen, Hilden, Germany), quality control and quantification measurement (Nanodrop Technologies, USA), ultrasonic fragmentation (Covaris, USA), library construction and target enrichment (SureSelect, Agilent Technologies, USA; Illumina, San Diego, USA) were conducted according to the manufacturers' protocols. High-throughput targeted measurement of gene mutations were performed on an lon torrent MiSeq/HiSeq (Illumina) sequencer or PGM ${ }^{\mathrm{TM}}$ (Life Technologies) platform with an average sequencing depth of 800x. The custom-designed panel consisted of 112 potentially mutated genes involved in hematological disorders which are related to the following functional categories: signaling pathways, epigenetic regulators, transcription factors, spliceosomes, cohesin complex, tumor suppressors, NPM1 and others. Single nucleotide variants (SNVs) and short fragment indels in protein coding sequence (CDS) were analyzed by using lon 
Reporter $^{\mathrm{TM}}$ and Variant Reporter pipelines and annotated referencing to the dbSNP, 1000 Genomes, Polyphen-2 and COSMIC databases. NPM1 (exon12), FLT3-ITD, and potential complex indels in CEBPA (TAD and bZIP domains) were additionally examined by the PCR followed by direct sequencing as previously reported.(30-32)

\section{Statistical analysis}

Descriptive statistics were presented as "median (range)" for non-normally distributed variables and "frequency (incidence)" for categorical variables. The $\chi 2$ test and Mann-Whitney $U$ test were used to calculate statistical significance for associations between coexisting mutations and MICM features. To extract independent factors, those with a $P$-value $<0.15$ were included as covariates in multivariate Logistic model using the forward stepwise selection procedure. All calculations were performed applying IBM SPSS v25.0 for Windows. In all analyses, $P$-values $<0.05$ were considered statistically significant. The Microsoft Excel 2013, Graphpad Prism 7.03, and Circos-0.69-9 software were also used for figure plotting.

\section{Results}

\section{FAB subtypes of NPM1 ${ }^{\text {mut }} A M L$}

In this study, we picked out 238 patients with NPM1mut AML for our purpose analysis. The study cohort consisted of 105 males and 133 females, with a median age of 49 (range 15-81) years. The most common FAB subtypes of $N P M 7^{\mathrm{mut}} A M L$ were AML-M5 (59.7\%), followed by M2 (17.6\%) and M4 (15.5\%), similar to other findings. $(8,9)$ According to FLT3-ITD, M2 is more common in NPM1 mut $_{\text {/FLT3-ITD }}^{(-)}$group [34/143 (23.8\%) vs. 8/95 (8.4\%); $P=0.002$ ], while M5 is slightly more common in $N P M 1^{\text {mut }}$ FLT3-ITD $^{(+)}$group [64/95 (67.4\%) vs. 78/143 (54.5\%); $\left.P=0.048\right]$, as shown in Fig. 1a.

\section{The expression incidence of CD34 and CD7 in NPM1 ${ }^{\text {mut }} /$ FLT3-ITD $^{(+)}$group were higher than in NPM1 ${ }^{\text {mut }} /$ FLT3-ITD $^{(-)} \mathrm{group}$}

As per literature(33), The leukemic blasts at the initial diagnosis could be divided into leukemic myeloid blasts and leukemic immature monocyte populations, with the latter detected in about $50 \%$ cases and mostly in M4 or M5 morphologic subtypes. The leukemic myeloid blasts recurred when AML relapsed, while leukemic immature monocyte populations often disappeared, indicating that the leukemic myeloid blasts may enrich more LSCs which serve as source of disease relapse. Consequently, in the description of baseline FCM characteristic, we only analyzed the antigen expression aspects of leukemic myeloid cells. In entire NPM1 ${ }^{\text {mut }}$ cohort, the antigens positively expressed at an incidence of 80\% or more were CD117 (211/235, 89.8\%), CD13 (207/233, 88.8\%), CD33 (233/233, 100\%), CD123 (230/232, 99.1\%) and CD38 (209/233, 89.7\%). The positive incidence of CD34 and TDT were 31.5\% (74/235) and 6.9\% (16/231), respectively. The positive incidence of HLA-DR was 67.4\% (157/233) and MPO was 74.9\% (57/227). CD7 was positively expressed in 43.1\% (94/218), CD19 in 3.5\% (8/228) and CD79a in 0.4\% $(1 / 227)$ cases, as shown in Fig. 1b. According to FLT3-ITD, the positive incidence of CD34 and CD7 in NPM1mut/FLT3-ITD ${ }^{(+)}$ were significantly higher than in NPM1 ${ }^{\text {mut }} / F L T 3-$ ITD $^{(-)}$group (CD34: 47.9\% vs. 20.6\%, P< 0.001; CD7: 61.5\% vs. 29.9\%, P< 0.001 ), while the positive incidence of other antigens were not different between the two genotypic groups.

\section{Chromosomal karyotypes in NPM1 ${ }^{\text {mut }} \mathrm{AML}$}

Of all 238 patients with NPM7 ${ }^{\text {mut }}, 234$ patients had evaluable metaphases, in whom 208 (88.9\%) were normal karyotypes (NK) and $26(11.1 \%)$ were abnormal karyotypes. Among 143 cases with NPM7mut $/$ FLT3-ITD ${ }^{(-)}, 140$ had evaluable metaphases, with 131 cases in the intermediate-risk group (including 121 cases NK; 10 cases intermediate-risk abnormal karyotype, Fig. 1c), 5 cases in the favorable-risk group [including 4 cases t(8;21)(q22;q22); 1 case inv (16) (p13q22)] and 4 cases in the adverse-risk group (including 1 case complex karyotype, monosomy karyotype, $t(6 ; 9)(p 23 ; q 34), t(8 ; 9 ; 22)$ (q24;q34;q11.2) for each]. Among 95 cases with $N P M 7^{\text {mut }} / F L T 3-$ ITD $^{(-)}, 94$ had evaluable metaphases, with all of them in the intermediate-risk group (including 87 cases NK; 7 cases intermediate-risk abnormal karyotype, Fig. 1c) and none in the favorable- or adverse-risk group. There was no difference in the distribution of both NK and intermediate-risk abnormal karyotype between $N P M 1^{\text {mut }} / F L T 3-I T D^{(-)}$and $N P M 1^{\text {mut }} / F L T 3-I_{T D}^{(+)}$group $(P=0.144$ and 0.930 , respectively), while the 
favorable- and adverse-risk karyotypes were only enriched in the NPM1 ${ }^{\text {mut }} / F L T 3-$ ITD $^{(-)}$group and not in the NPM1 ${ }^{\text {mut }} / F L T 3-$ $\operatorname{ITD}^{(+)}$group $(6.4 \%$ vs. $0 \% ; P=0.031)$. No correlation was found between other coexisting gene mutations and abnormal karyotypes (all $P>0.05$, data not shown). No KMT2A (MLL) or TP53 rearrangement was identified in $206 N P M 7^{\text {mut }}$ patients with available FISH data.

\section{NPM1 mut loci, types and co-mutation patterns}

In entire NPM1mut cohort, totaling 240 NPM1 mutant events were identified, among whom 230 (230/240, 95.8\%) were out-offrame indels. Ten cases $(10 / 240,4.2 \%)$ were detected to habour $N P M 7^{\text {mut }}$ missense events, i.e., 3 cases with c.578A > $\mathrm{G} \rightarrow$ p.K193R, 2 with c.676G $>$ A $\rightarrow$ p.E226K and 5 with c.733G $>$ C $\rightarrow$ p.E245Q. All these missense codons did not disrupt any of the tryptophan residues W288 and W290 which are indispensible for the nucleolar localization signal (NoLS). Furthermore, all but one of these missense mutations $(9 / 10,90.0 \%)$ were accompanied by an AML subtype-defining recurrent cytogenetic or molecular abnormality, with 7 cases in the favorable risk and 2 in adverse risk (Table 1).

Table 1

AML subtype-defining cytogenetic or molecular abnormalities accompanied by $N P M 7^{\text {mut }}$ missense mutations

\begin{tabular}{|c|c|}
\hline NPM1 ${ }^{\text {mut }}$ missense & AML subtype-defining cytogenetic or molecular abnormalities \\
\hline c.578A $>\mathrm{G} \rightarrow$ p.K193R & $t(8 ; 21)(q 22 ; q 22),-Y$ \\
\hline c.578A $>\mathrm{G} \rightarrow$ p.K193R & $\operatorname{inv}(16)(p 13 q 22)$ \\
\hline c.578A $>\mathrm{G} \rightarrow$ p.K193R & $t(6 ; 9)(p 23 ; q 34)$ \\
\hline c. $676 \mathrm{G}>\mathrm{A} \rightarrow \mathrm{p} . \mathrm{E} 226 \mathrm{~K}$ & $N P M 7^{\text {mut }}\left[\mathrm{p} . \mathrm{W} 288 \mathrm{Cfs}{ }^{\star 12}\right]$ \\
\hline c. $676 \mathrm{G}>\mathrm{A} \rightarrow \mathrm{p} . \mathrm{E} 226 \mathrm{~K}$ & CEBPA ${ }^{\mathrm{dm}}$ [p.E309_D322indelsGQTQQKVLELTS; p.D80Gfs] \\
\hline c.733G $>$ C $\rightarrow$ p.E245Q & $t(8 ; 21)(q 22 ; q 22)$ \\
\hline c.733G $>$ C $\rightarrow$ p.E245Q & Complex: $+8,-14, \mathrm{i}(17)(\mathrm{q} 10),+2 \sim 3 \mathrm{mar}$ \\
\hline c.733G $>$ C $\rightarrow$ p.E245Q & $N P M 7^{\text {mut }}\left[\mathrm{p} . \mathrm{W} 288 \mathrm{Cfs}{ }^{\star 12}\right]$ \\
\hline c.733G > C $\rightarrow$ p.E245Q & $C E B P A^{\mathrm{dm}}[\mathrm{p} . \mathrm{K} 313 \mathrm{dupK} ; \mathrm{p} . \mathrm{Q} 87 \mathrm{X}]$ \\
\hline c.733G > C $\rightarrow$ p.E245Q & - \\
\hline Abbreviations: $N P M 1^{n}$ & t, NPM1 mutation; CEBPAdm, double allele mutation of $C E B P A$ \\
\hline
\end{tabular}

At least one co-mutation was detected in all $238 N P M 7^{\text {mut }}$ cases. Including $N P M 7^{\text {mut }}$, the median number of mutated genes per individual was $4.5(2-14)$, with $4.0(2-14)$ in the $N P M 7^{\text {mut }} / F L T 3-I T D^{(-)}$, insignificantly different from $5.0(2-10)$ in the $N P M 7^{\text {mut }} / F_{L T 3}$ ITD $^{(+)}$group $(P=0.378$, Fig. 2a). According to gene function categories, the order of incidence is as follows: signaling pathways (72.7\%), epigenetic regulators (71.4\%), tumor suppressors (31.9\%) and myeloid transcription factors (8.8\%, Fig. 2b). DNMT3A (104, 43.7\%), FLT3-ITD (95, 39.9\%) and $F A T 1$ (57, 23.9\%) represented the top three most frequently mutated genes (more details on relatively common genes with a mutation frequency $>5 \%$ are listed in Fig. 3 ). Analysis of gene-gene relationship across $N P M 7^{\text {mut }}$ coexisting mutations showed that FLT3-ITD and DNMT3A had significant accompaniment $(P=0.005)$, while FLT3-ITD was mutually exclusive of $F L T 3$-nonITD $(P<0.001), N R A S(P<0.001), P T P N 11(P$ $=0.017)$ and $I D H 1(P=0.005$, Fig. 4).

\section{Association between NPM1 ${ }^{\text {mut }}$ coexisting mutations and immunophenotypic markers}


Our results have shown that the expression of CD34 and CD7 is significantly associated with FLT3-ITD. Because NPM7mut AML mostly occurs in the context of NK, we hypothesize that the diversity of antigen expression in leukemia cells to a certain extent is determined by heterogeneity of coexisting mutations. In order to rule out the influence of abnormal karyotype on immunophenotype, as well in view of the deductively insufficient pathogenicity of NPM $7^{\text {mut }}$ missense mutations, only patients with NK and NPM $7^{\text {mut }}$ indels type were included for subsequent analysis. A total of 205 NPM $7^{\text {mut }}$ patients meeting the above conditions were submitted to the distributional crosstabulation between immunophenotypic markers and coexisting mutations. The significant results from $\chi 2$ test and multivariate analysis are shown in Table 2. Logistic analysis showed that, in entire NPM7 ${ }^{\text {mut }}$ cohort, FLT3-ITD was positively correlated with CD34 and CD7 ( $H R=5.29$ [95\% Cl 2.64-10.60], $P<0.001$; $H R=3.47$ [95\% Cl 1.79-6.73], $P<0.001)$. Ras-pathway mutation was positively correlated with HLA-DR expression $(H R=4.05$ [95\% Cl 1.70-9.63], $P=0.002)$ and negatively with MPO expression $(H R=0.18$ [95\% $\mathrm{Cl} 0.05-0.62], P=0.007)$ in entire $N P M 7^{\text {mut }}$ cohort. Stratified analysis indicated that this effect was only seen in NPM $7^{\text {mut }} / F L T 3-$ ITD $^{(-)}$group (HR and $P$ values are detailed in Table 2), but not in NPM1 ${ }^{\text {mut }} / F L T 3-$ ITD $^{(-)}$group. 
Table 2

Correlation between immunophenotypic markers with mutations of coexisting genes in NPM1 ${ }^{\text {mut }} A M L$

\begin{tabular}{|c|c|c|c|c|c|c|c|c|c|c|}
\hline \multicolumn{2}{|c|}{ Association } & \multicolumn{3}{|c|}{ NPM1 $7^{\mathrm{mut}} / F L T 3 \mathrm{ITD}^{(-)}$} & \multicolumn{3}{|c|}{ NPM1 $1^{\mathrm{mut}} /$ FLT3 TTD $^{(+)}$} & \multicolumn{3}{|c|}{ Entire cohort } \\
\hline & & $\chi 2 P$ & $\begin{array}{l}\mathrm{HR} \\
(95 \% \mathrm{Cl})\end{array}$ & $\mathrm{P}$ & $\chi 2 P$ & $\begin{array}{l}\mathrm{HR} \\
(95 \% \mathrm{Cl})\end{array}$ & $\mathrm{P}$ & $\chi 2 P$ & $\begin{array}{l}\mathrm{HR} \\
(95 \% \mathrm{Cl})\end{array}$ & $\mathrm{P}$ \\
\hline \multirow{3}{*}{$\begin{array}{l}\text { CD34 } \\
(\mathrm{N}= \\
202)\end{array}$} & ×FLT3-TD & NA & NA & NA & NA & NA & NA & $<0.001$ & $\begin{array}{l}5.29 \\
(2.64- \\
10.60)\end{array}$ & $<0.001$ \\
\hline & $\times D N M T 3 A$ & NS & NA & NA & 0.028 & $\begin{array}{l}2.60 \\
(1.00- \\
6.79)\end{array}$ & 0.051 & 0.026 & NA & NA \\
\hline & $\times$ TET2/IDH1 & NS & NA & NA & 0.005 & $\begin{array}{l}0.21 \\
(0.06- \\
0.71)\end{array}$ & 0.012 & 0.001 & $\begin{array}{l}0.26 \\
(0.11- \\
0.62)\end{array}$ & 0.002 \\
\hline \multirow{4}{*}{$\begin{array}{l}\text { CD7 } \\
(\mathrm{N}= \\
186)\end{array}$} & ×FLT3-ITD & NA & NA & NA & NA & NA & NA & $<0.001$ & $\begin{array}{l}3.47 \\
(1.79- \\
6.73)\end{array}$ & $<0.001$ \\
\hline & $\times D N M T 3 A$ & 0.008 & NA & NA & 0.007 & $\begin{array}{l}3.30 \\
(1.15- \\
9.46)\end{array}$ & 0.026 & $<0.001$ & NA & NA \\
\hline & $\begin{array}{l}\times D N M T 3 A- \\
\text { R882 }\end{array}$ & 0.002 & $\begin{array}{l}3.93 \\
(1.61- \\
9.59)\end{array}$ & 0.003 & 0.009 & NA & NA & $<0.001$ & $\begin{array}{l}3.59 \\
(1.80- \\
7.16)\end{array}$ & $<0.001$ \\
\hline & $\times T E T 2 / I D H 1$ & 0.048 & NA & NA & 0.001 & $\begin{array}{l}0.18 \\
(0.05- \\
0.60)\end{array}$ & 0.005 & NS & $\begin{array}{l}0.30 \\
(0.14- \\
0.62)\end{array}$ & 0.001 \\
\hline \multirow{3}{*}{$\begin{array}{l}\text { HLA- } \\
\text { DR } \\
(\mathrm{N}= \\
200)\end{array}$} & $\begin{array}{l}\times \text { Ras } \\
\text { pathways }\end{array}$ & 0.002 & $\begin{array}{l}3.83 \\
(1.40- \\
10.46)\end{array}$ & 0.009 & 0.055 & NA & NA & $<0.001$ & $\begin{array}{l}4.05 \\
(1.70- \\
9.63)\end{array}$ & 0.002 \\
\hline & $\begin{array}{l}\times D N M T 3 A- \\
\text { R882 }\end{array}$ & $\begin{array}{l}< \\
0.001\end{array}$ & $\begin{array}{l}26.77 \\
(3.44- \\
208.46)\end{array}$ & 0.002 & $<0.001$ & $\begin{array}{l}8.65 \\
(2.28- \\
32.89)\end{array}$ & 0.002 & $<0.001$ & $\begin{array}{l}13.41 \\
(4.56- \\
39.45)\end{array}$ & $<0.001$ \\
\hline & $\times$ TET2/IDH1 & NS & NA & NA & 0.002 & $\begin{array}{l}0.26 \\
(0.09- \\
0.78)\end{array}$ & 0.016 & 0.046 & NA & NA \\
\hline MPO & $\times K R A S$ & 0.002 & $\begin{array}{l}0.13 \\
(0.03- \\
0.56)\end{array}$ & 0.006 & NS & NA & NA & 0.003 & $\begin{array}{l}0.18 \\
(0.05- \\
0.62)\end{array}$ & 0.007 \\
\hline \multirow[t]{3}{*}{$\begin{array}{l}(\mathrm{N}= \\
196)\end{array}$} & $\times D N M T 3 A$ & 0.003 & NA & NA & 0.071 & NA & NA & $<0.001$ & $\begin{array}{l}0.35 \\
(0.17- \\
0.70)\end{array}$ & 0.003 \\
\hline & $\begin{array}{l}\times D N M T 3 A- \\
\text { R882 }\end{array}$ & 0.002 & $\begin{array}{l}0.27 \\
(0.10- \\
0.74)\end{array}$ & 0.011 & NS & NA & NA & 0.001 & NA & NA \\
\hline & $\times T E T 2 / I D H 1$ & 0.040 & NA & NA & 0.021 & $\begin{array}{l}4.32 \\
(1.16- \\
16.15)\end{array}$ & 0.029 & 0.001 & $\begin{array}{l}3.52 \\
(1.48- \\
8.38)\end{array}$ & 0.004 \\
\hline $\begin{array}{l}\text { APL- } \\
\text { like }\end{array}$ & $\begin{array}{l}\times \text { Ras } \\
\text { pathways }\end{array}$ & 0.008 & $\begin{array}{l}0.32 \\
(0.11- \\
0.96)\end{array}$ & 0.041 & 0.025 & NA & NA & $<0.001$ & $\begin{array}{l}0.22 \\
(0.08- \\
0.57)\end{array}$ & 0.002 \\
\hline
\end{tabular}

198)

Abbreviations: NS, no significance; NA, not applicable 


\begin{tabular}{|c|c|c|c|c|c|c|c|c|c|}
\hline $\begin{array}{l}\times D N M T 3 A- \\
\text { R882 }\end{array}$ & $\begin{array}{l}< \\
0.001\end{array}$ & NA & NA & $<0.001$ & $\begin{array}{l}0.04 \\
(0.01- \\
0.36)\end{array}$ & 0.004 & $<0.001$ & $\begin{array}{l}0.02 \\
(0.00- \\
0.18)\end{array}$ & $<0.001$ \\
\hline$\times T E T 2 / I D H 1$ & NS & NA & NA & $<0.001$ & $\begin{array}{l}6.73 \\
(1.83- \\
24.78)\end{array}$ & 0.004 & 0.008 & $\begin{array}{l}2.26 \\
(1.07- \\
4.78)\end{array}$ & 0.033 \\
\hline
\end{tabular}

Abbreviations: NS, no significance; NA, not applicable

DNMT3A-R882 was positively correlated with CD7 and HLA-DR expression $(H R=3.59$ [95\% $\mathrm{Cl} 1.80-7.16], P<0.001 ; H R=$ 13.41 [95\% Cl 4.56-39.45], $P<0.001)$, and DNMT3A mutation negatively with MPO expression $(H R=0.35$ [95\% $\mathrm{Cl} 1.48-8.38]$, $P=0.004)$. Stratified analysis according to FLT3-ITD status indicated that the independent correlation of DNMT3A mutation (especially DNMT3A-R882) with CD7 and with HLA-DR expression was significant both in NPM1 ${ }^{\text {mut }} / F_{L L T 3}-$ ITD $^{(+)}$and in $N P M 7^{\text {mut }} / F L T 3-$ ITD $^{(-)}$groups (HR and $P$ values are detailed in Table 2). TET2/IDH1 mutations were negatively correlated with CD34 and CD7 expression $(H R=0.26$ [95\% Cl 0.11-0.62], $P=0.002 ; H R=0.30$ [95\% Cl 0.14-0.62], $P=0.001)$, and positively with MPO expression $(H R=3.52$ [95\% $\mathrm{Cl} 1.48-8.38$ ], $P=0.004)$. Stratified analysis indicated the above effects to be prominent

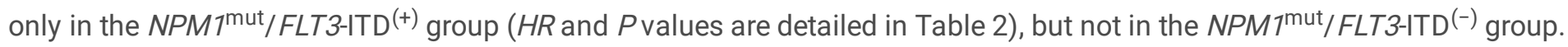
There were no significant correlations between $N P M 7^{\text {mut }}$ coexisting mutations with other antigen expressions.

We finally analyzed association of $N P M 7^{\text {mut }}$ coexisting mutations with APL-like phenotype $\mathrm{CD} 34^{(-)} / \mathrm{HLA}^{-\mathrm{DR}} \mathrm{R}^{(-)} / \mathrm{MPO}^{(\mathrm{str}+)}$, which has been reportedly to predict a presence of TET2/IDH1 mutation.(12) In entire cohort, mutations of Ras-pathway, DNMT3A-R882 and TET2/IDH1 were each significantly linked with APL-like phenotype. When layered by FLT3-ITD, in NPM7 ${ }^{\text {mut }} / F L T 3$-ITD ${ }^{(-)}$group, only Ras-pathway mutation presented an association with APL-like phenotype $(H R=0.32[95 \% \mathrm{Cl}$ 0.11-0.96], $P=0.041)$. Comparatively, negative correlation of DNMT3A-R882 $(H R=0.04[95 \% \mathrm{Cl} 0.01-0.36], P=0.004)$ and positive correlation of $T E T 2 / I D H 1$ mutation $(H R=6.73$ [95\% $\mathrm{Cl} 1.83-24.78], P=0.004)$ with this phenotype were seen only in

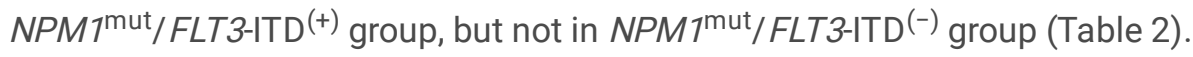

\section{Discussion}

Previous studies concerning prognosis of $N P M 7^{\text {mut }} A M L$ often depicted its mutational type as indels, and there was little information about other types of NPM1 ${ }^{\text {mut }}$. In our cohort of $238 N P M 7^{\text {mut }}$ patients, 240 NPM1 mutant events were identified, therein the vast majority $(232,99.1 \%)$ were indels type, all of them deranging the tryptophan residues W288 and W290 which are indispensably responsible for NoLS.(2) Ten NPM1 ${ }^{\text {mut }}$ missense mutations were clustered in NPM1 ${ }^{\text {mut }} /$ FLT3-ITD $^{(-)} \mathrm{group}$, and none of them disrupt the NoLS loci, nor were they involved in NPM1 post-translational modification sites(3). Moreover, all except one (9,90.0\%) missense mutations were accompanied with an AML subtype-defining favorable- and adverse-risk cytogenetic or molecular abnormality, indicating that $N P M 7^{\text {mut }}$ missense may be insufficient to drive leukemogenesis and necessitate other well-characterized pathomechanism. When we mention theme of NPM7 ${ }^{\text {mut }}$ prognostication, we will be referring to mutations exclusively in the context of its indels type, rather than including missense mutations with relatively rarity and inadequate pathogenicity.

In this study, NK reached $\sim 90 \%$ in entire $N P M 7^{\text {mut }}$ cohort with analyzable metaphases and accounted for $84.6 \%$ in NPM1 mut $^{\text {F } F L T 3-I T D^{(-)}}$group, similar with the finding of $82.4 \%$ in a large sample survey.(13) Moreover, the recurrent cytogenetic translocations were uncommon and FISH did not detect any of KMT2A (MLL) translocations or TP53 deletions, implying the leukemogenesis of frameshift $N P M 7^{\text {mut }}$ not relying on chromosomal abnormality. Nonetheless, all $N P M 7^{\text {mut }}$ indels arised together with coexisting mutations, especially those affecting epigenetic regulators and signaling pathways, which points to the necessity of interactivity of $N P M 7^{\text {mut }}$ with other genetic lesions to promote leukemic overt occurrence. The favorable- and

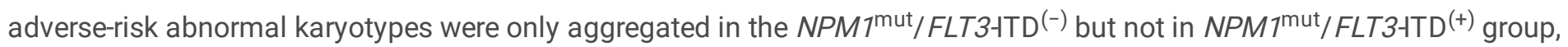
suggestive of a mutual exclusivity or pathogenic independence between FLT3-ITD and these karyotypes in NPM1 ${ }^{\mathrm{mut}}$ AML. 


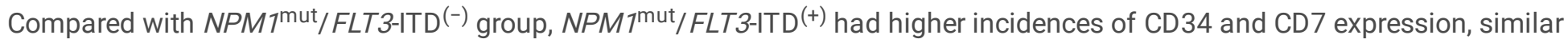
to other reports.(34) FCM immunophenotyping is not only used in the differential diagnosis of AML, but also has prognostic value. In terms of an individual immunomarker, CD34(+) in NPM7 ${ }^{\text {mut }}$ AML was associated with a poor prognosis. $(11,15)$ CD123 was only expressed in leukemia and other neoplastic cells, but hardly in normal hematopoietic cells.(35) The percentage of $\mathrm{CD}_{123^{(+)}}$cells in $N P M 7^{\text {mut }}$ patients divided by cutoff $52 \%$ was also reported to predict prognosis.(36) Going forward, combination of multiple aspects of antigen expression can more potently predict survival. Especially, the $\mathrm{CD} 34^{(+)} / \mathrm{CD} 38^{(-)} / \mathrm{CD} 123^{(+)}$which represents a LSC phenotype has prognostic relevance.(16) In addition, most LSC phenotypes also present cross-lineage, antigen overexpression or asynchronous expression phenomena.(16) In this study, the positive incidences of stem cell antigen CD34 and cross-lineage antigen CD7 were higher in NPM1 ${ }^{\text {mut }} /$ FLT3-ITD $^{(+)}$group, which may be implied to encompass more LSCs at initial presentation. LSCs are in the relatively silent cell cycle G0 phase and highly express the drug-resistant efflux transporter P-glycoprotein (PGP) or multidrug-resistant protein (MDR1).(11, 37) Chen CY et al. (17) clustered immunophenotyping in 94 NPM ${ }^{\text {mut }}$ patients and divided them into two categories according to CD34, CD7 and HLA-DR, showing that the prognosis of type- $\llbracket$ class characterized by $\mathrm{CD} 34^{(+)} / \mathrm{HLA}-\mathrm{DR}{ }^{(+)} / \mathrm{CD} 7^{(+)}$was significantly poorer versus the type- $₫$ class $C D 34^{(-)} / C D 7^{(-)}$. However, their results may be affected by the biased distribution of concurrent FLT3ITD, which has a positive correlation with CD34 and CD7. Due to limited number of cases, it was not clear whether the

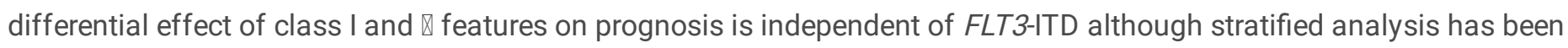
carried out.

We investigated the relationship between $N P M 1^{\text {mut }}$ coexisting mutations and immunophenotypic markers. In general, there is a distributional association of signaling and methylating mutations with CD34, CD7, HLA-DR and MPO expression. The

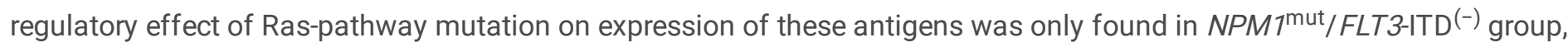
but not in NPM7mut $/ F L T 3-I T D^{(+)}$group, partly owing to the reciprocal exclusivity of FLT3-ITD with Ras-pathway mutation. Although both involved in methylation regulation, DNMT3A mutation was positively correlated with the expression of CD34, CD7 and HLA-DR, while TET2/IDH1 mutation negatively with these antigens. By contrast, DNMT3A mutation was negatively correlated with MPO expression, while TET2/IDH1 mutation positively with MPO expression. As per FLT3-ITD status, in NPM1 ${ }^{\text {mut }} /$ FLT3-ITD $^{(-)}$group, specific DNMT3A-R882 was independently correlated with CD7, HLA-DR and MPO expression, while TET2/IDH1 mutation showed no correlation with the expression of these antigens. In NPM1 ${ }^{\text {mut } / F L T 3-I T D ~}{ }^{(+)}$group, DNMT3A and TET2/IDH1 mutations were each independently related to, and resultingly may jointly determine the trend of expression of CD34, CD7 and HLA-DR.

In NPM1 ${ }^{\text {mut }} /$ FLT3-ITD $^{(-)}$group, Ras-pathway mutation and DNMT3A-R882 were positively correlated with the expression of monocyte marker HLA-DR, and negatively with myeloid marker MPO, which is linked to the morphological association of FAB typing in monocytic differentiation (M4/M5) or granulocytic differentiation (M2). Comparatively, in NPM7 mut $/ F L T 3-I T D(+)^{(+)}$ group, although TET2/IDH1 mutation was negatively correlated with HLA-DR expression, the more commonly coexisting DNMT3A-R882 which was positively correlated with HLA-DR expression was in concordance with a more frequent M4/M5 morphology in this genotypic subset.

Mason EF et al.(12) had analyzed the myeloid blast populations excluding monocytic differentiation in NPM1mut patients. Nearly half of cases $(48 \%)$ had an APL-like phenotype represented by CD34(-)/HLA-DR ${ }^{(-)} / \mathrm{MPO}^{(\mathrm{str}+)}$, which could predict a presence of TET2 or IDH1/2 mutations, a result in line with our findings. Moreover, they demonstrated the APL-like phenotype to beneficially impact RFS and OS, and its combination with TET2 or IDH1/2 coexisting mutations to be more explicit to refine prognostic subgroups. The present study extends those findings. In addition to TET2 or IDH1/2, we also showed an independent association of both Ras-pathway mutations and DNMT3A-R882 with APL-like phenotype, and this relationship

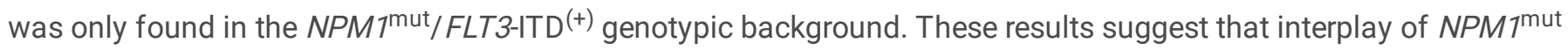
coexisting genetic lesions may jointly determine the trend of antigen expression, partly explaining the immunophenotypic heterogeneity in $N P M 7^{\text {mut }} \mathrm{AML}$. 
To sum up, NPM1 ${ }^{\text {mut }}$ missense mutations may be of leukemogenic insufficiency, and largely rely on other well-defined pathomechanism to the development of leukemia. Correlation of coexisting mutations with antigen expressions in NPM $7^{\text {mut }}$ AML partly explains the immunophenotypic diversity. Comprehensively evaluating FCM immunophenotype and NGS landscape of genetic lesions allows us to gain insight into the clinicopathological heterogeneity for this distinct AML entity.

\section{Declarations}

\section{Author Contributions:}

B.W. collected clinical-laboratory data and wrote the article; B.Y. and W.W. performed statistical analysis; X.L. performed sequencing and interpreted mutational data; Hq.L. was responsible for study conception and design. All co-authors have read and approved the final manuscript.

\section{Conflict of interest statement:}

The authors declare that they have no conflict of interests.

\section{Acknowledgements:}

We thank all participants in this clinical study and all coworkers in our laboratory for their excellent technical assistance and providing data.

\section{Availability of data and materials:}

The datasets used and/or analyzed during the current study are available from the corresponding author on reasonable request.

\section{References}

1. Kunchala P, Kuravi S, Jensen R, et al. When the good go bad: Mutant NPM1 in acute myeloid leukemia. Blood Rev, 2018, 32(3): 167-83. doi: 10.1016/j.blre.2017.11.001.

2. Brodska B, Sasinkova M, Kuzelova K. Nucleophosmin in leukemia: Consequences of anchor loss. Int J Biochem Cell Biol, 2019, 111: 52-62. doi: 10.1016/j.biocel.2019.04.007.

3. Heath EM, Chan SM, Minden MD, et al. Biological and clinical consequences of NPM1 mutations in AML. Leukemia, 2017, 31(4): 798-807. doi: 10.1038/leu.2017.30.

4. Bullinger L, Dohner K, Dohner H. Genomics of Acute Myeloid Leukemia Diagnosis and Pathways. J Clin Oncol, 2017, 35(9): 934-46. doi: 10.1200/JC0.2016.71.2208.

5. Falini B, Nicoletti I, Bolli N, et al. Translocations and mutations involving the nucleophosmin (NPM1) gene in lymphomas and leukemias. Haematologica, 2007, 92(4): 519-32. doi: 10.3324/haematol.11007.

6. Falini B, Mecucci C, Tiacci E, et al. Cytoplasmic nucleophosmin in acute myelogenous leukemia with a normal karyotype. N Engl J Med, 2005, 352(3): 254-66. doi: 10.1056/NEJMoa041974.

7. Cancer Genome Atlas Research N, Ley TJ, Miller C, et al. Genomic and epigenomic landscapes of adult de novo acute myeloid leukemia. N Engl J Med, 2013, 368(22): 2059-74. doi: 10.1056/NEJMoa1301689.

8. Schnittger S, Schoch C, Kern W, et al. Nucleophosmin gene mutations are predictors of favorable prognosis in acute myelogenous leukemia with a normal karyotype. Blood, 2005, 106(12): 3733-9. doi: 10.1182/blood-2005-06-2248.

9. Wilson CS, Davidson GS, Martin SB, et al. Gene expression profiling of adult acute myeloid leukemia identifies novel biologic clusters for risk classification and outcome prediction. Blood, 2006, 108(2): 685-96. doi: 10.1182/blood-2004-124633. 
10. Chen W, Konoplev S, Medeiros LJ, et al. Cuplike nuclei (prominent nuclear invaginations) in acute myeloid leukemia are highly associated with FLT3 internal tandem duplication and NPM1 mutation. Cancer, 2009, 115(23): 5481-9. doi:

10.1002/cncr.24610.

11. Zeijlemaker W, Kelder A, Wouters R, et al. Absence of leukaemic CD34(+) cells in acute myeloid leukaemia is of high prognostic value: a longstanding controversy deciphered. Br J Haematol, 2015, 171(2): 227-38. doi: 10.1111/bjh.13572.

12. Mason EF, Kuo FC, Hasserjian RP, et al. A distinct immunophenotype identifies a subset of NPM1-mutated AML with TET2 or IDH1/2 mutations and improved outcome. Am J Hematol, 2018, 93(4): 504-10. doi: 10.1002/ajh.25018.

13. Angenendt L, Rollig C, Montesinos P, et al. Chromosomal Abnormalities and Prognosis in NPM1-Mutated Acute Myeloid Leukemia: A Pooled Analysis of Individual Patient Data From Nine International Cohorts. J Clin Oncol, 2019, 37(29): 263242. doi: $10.1200 / J C 0.19 .00416$.

14. Vassiliou GS, Cooper JL, Rad R, et al. Mutant nucleophosmin and cooperating pathways drive leukemia initiation and progression in mice. Nat Genet, 2011, 43(5): 470-5. doi: 10.1038/ng.796.

15. Dang H, Chen Y, Kamel-Reid S, et al. CD34 expression predicts an adverse outcome in patients with NPM1-positive acute myeloid leukemia. Hum Pathol, 2013, 44(10): 2038-46. doi: 10.1016/j.humpath.2013.03.007.

16. van Rhenen A, Moshaver B, Kelder A, et al. Aberrant marker expression patterns on the CD34+CD38- stem cell compartment in acute myeloid leukemia allows to distinguish the malignant from the normal stem cell compartment both at diagnosis and in remission. Leukemia, 2007, 21(8): 1700-7. doi: 10.1038/sj.leu.2404754.

17. Chen CY, Chou WC, Tsay W, et al. Hierarchical cluster analysis of immunophenotype classify AML patients with NPM1 gene mutation into two groups with distinct prognosis. BMC Cancer, 2013, 13: 107. doi: 10.1186/1471-2407-13-107.

18. Metzeler $\mathrm{KH}$, Herold $\mathrm{T}$, Rothenberg-Thurley $\mathrm{M}$, et al. Spectrum and prognostic relevance of driver gene mutations in acute myeloid leukemia. Blood, 2016, 128(5): 686-98. doi: 10.1182/blood-2016-01-693879.

19. Metzeler KH, Maharry K, Radmacher MD, et al. TET2 mutations improve the new European LeukemiaNet risk classification of acute myeloid leukemia: a Cancer and Leukemia Group B study. J Clin Oncol, 2011, 29(10): 1373-81. doi:

10.1200/JCO.2010.32.7742.

20. Tian $X, X u$ Y, Yin J, et al. TET2 gene mutation is unfavorable prognostic factor in cytogenetically normal acute myeloid leukemia patients with NPM1+ and FLT3-ITD - mutations. Int J Hematol, 2014, 100(1): 96-104. doi: 10.1007/s12185-0141595-x.

21. Marcucci G, Maharry K, Wu YZ, et al. IDH1 and IDH2 gene mutations identify novel molecular subsets within de novo cytogenetically normal acute myeloid leukemia: a Cancer and Leukemia Group B study. J Clin Oncol, 2010, 28(14): 234855. doi: 10.1200/JCO.2009.27.3730.

22. Paschka P, Schlenk RF, Gaidzik VI, et al. IDH1 and IDH2 mutations are frequent genetic alterations in acute myeloid leukemia and confer adverse prognosis in cytogenetically normal acute myeloid leukemia with NPM1 mutation without FLT3 internal tandem duplication. J Clin Oncol, 2010, 28(22): 3636-43. doi: 10.1200/JC0.2010.28.3762.

23. Bacher U, Haferlach T, Schoch C, et al. Implications of NRAS mutations in AML: a study of 2502 patients. Blood, 2006, 107(10): 3847-53. doi: 10.1182/blood-2005-08-3522.

24. Boddu P, Kantarjian H, Borthakur G, et al. Co-occurrence of FLT3-TKD and NPM1 mutations defines a highly favorable prognostic AML group. Blood Adv, 2017, 1(19): 1546-50. doi: 10.1182/bloodadvances.2017009019.

25. Dufour A, Schneider F, Hoster E, et al. Monoallelic CEBPA mutations in normal karyotype acute myeloid leukemia: independent favorable prognostic factor within NPM1 mutated patients. Ann Hematol, 2012, 91(7): 1051-63. doi: 10.1007/s00277-012-1423-4.

26. Tallman MS, Wang ES, Altman JK, et al. Acute Myeloid Leukemia, Version 3.2019, NCCN Clinical Practice Guidelines in Oncology. J Natl Compr Canc Netw, 2019, 17(6): 721-49. doi: 10.6004/jnccn.2019.0028.

27. Dohner H, Estey E, Grimwade D, et al. Diagnosis and management of AML in adults: 2017 ELN recommendations from an international expert panel. Blood, 2017, 129(4): 424-47. doi: 10.1182/blood-2016-08-733196. 
28. Vardiman JW, Thiele J, Arber DA, et al. The 2008 revision of the World Health Organization (WHO) classification of myeloid neoplasms and acute leukemia: rationale and important changes. Blood, 2009, 114(5): 937-51. doi:

10.1182/blood-2009-03-209262.

29. International Standing Committee on Human Cytogenetic Nomenclature., Shaffer LG, McGowan-Jordan J, et al. ISCN 2013 : an international system for human cytogenetic nomenclature (2013). Basel: Karger; 2013. vi, 140 p. , 1 folded sheet p.

30. Lin LI, Chen CY, Lin DT, et al. Characterization of CEBPA mutations in acute myeloid leukemia: most patients with CEBPA mutations have biallelic mutations and show a distinct immunophenotype of the leukemic cells. Clin Cancer Res, 2005, 11(4): 1372-9. doi: 10.1158/1078-0432.CCR-04-1816.

31. Rau R, Brown P. Nucleophosmin (NPM1) mutations in adult and childhood acute myeloid leukaemia: towards definition of a new leukaemia entity. Hematol Oncol, 2009, 27(4): 171-81. doi: 10.1002/hon.904.

32. Kiyoi H, Naoe T, Nakano Y, et al. Prognostic implication of FLT3 and N-RAS gene mutations in acute myeloid leukemia. Blood, 1999, 93(9): 3074-80. doi, https://www.ncbi.nIm.nih.gov/pubmed/10216104

33. Zhou Y, Moon A, Hoyle E, et al. Pattern associated leukemia immunophenotypes and measurable disease detection in acute myeloid leukemia or myelodysplastic syndrome with mutated NPM1. Cytometry B Clin Cytom, 2019, 96(1): 67-72. doi: 10.1002/cyto.b.21744.

34. Kumar D, Mehta A, Panigrahi MK, et al. DNMT3A (R882) mutation features and prognostic effect in acute myeloid leukemia in Coexistent with NPM1 and FLT3 mutations. Hematol Oncol Stem Cell Ther, 2018, 11(2): 82-9. doi: 10.1016/j.hemonc.2017.09.004.

35. Al-Mawali A, Gillis D, Lewis I. Immunoprofiling of leukemic stem cells CD34+/CD38-/CD123+ delineate FLT3/ITD-positive clones. J Hematol Oncol, 2016, 9(1): 61. doi: 10.1186/s13045-016-0292-z.

36. Nomdedeu J, Bussaglia E, Villamor N, et al. Immunophenotype of acute myeloid leukemia with NPM mutations: prognostic impact of the leukemic compartment size. Leuk Res, 2011, 35(2): 163-8. doi: 10.1016/j.leukres.2010.05.015.

37. Gentles AJ, Plevritis SK, Majeti R, et al. Association of a leukemic stem cell gene expression signature with clinical outcomes in acute myeloid leukemia. JAMA, 2010, 304(24): 2706-15. doi: 10.1001/jama.2010.1862.

\section{Figures}




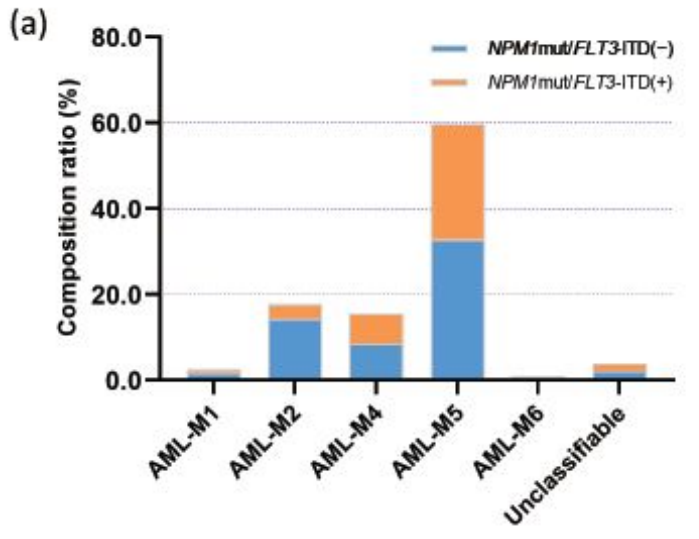

(b)

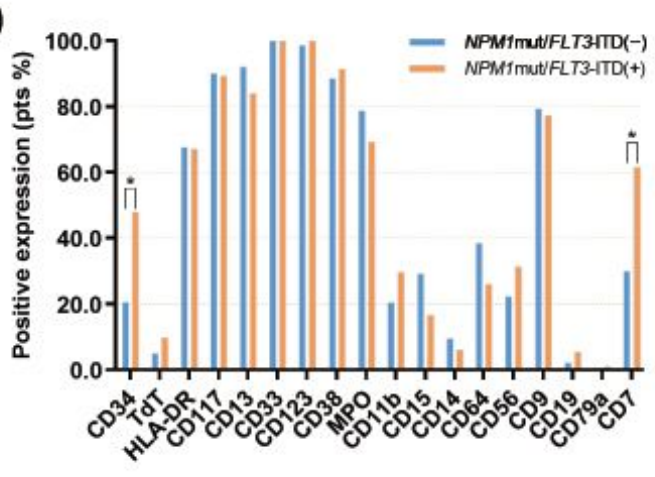

(c)

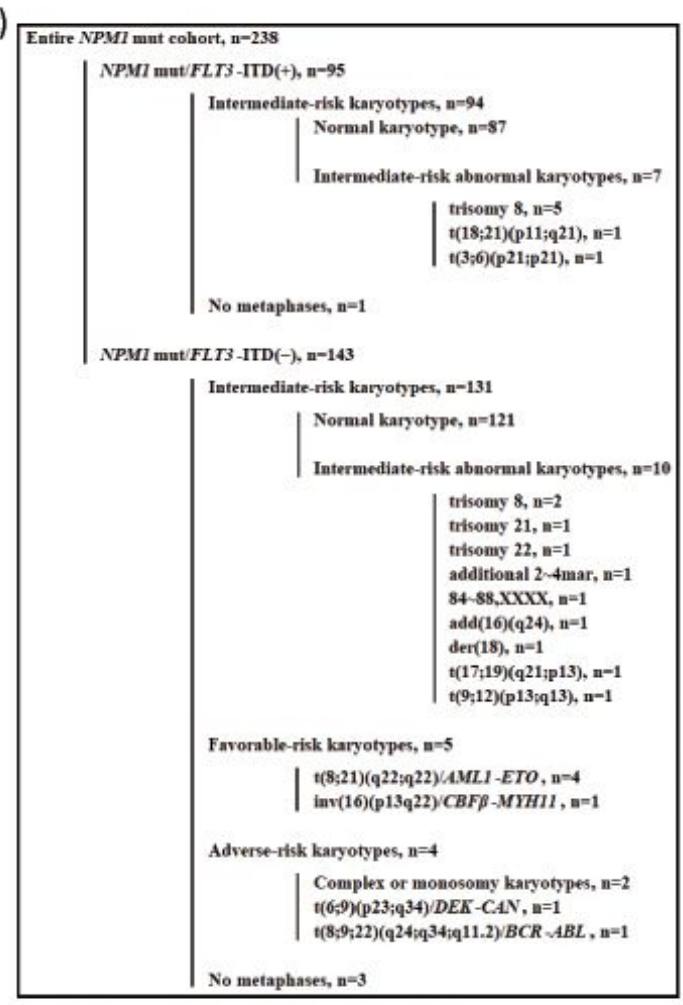

\section{Figure 1}

(a) Composition ratio of morphologic FAB subtypes in 238 patients with NPM1mut AML (by FLT3-ITD). (b) Positive expression rate of antigens on FCM (by FLT3-ITD); *, P<0.05. (c) Conventional banding karyotype (by FLT3-ITD and cytogenetic risk classification).

(a)

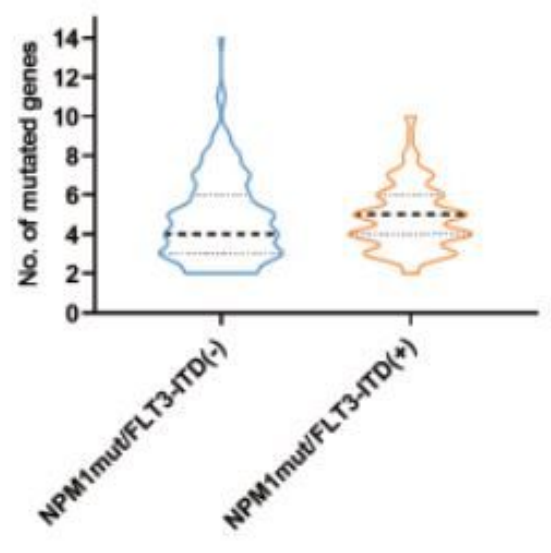

(b)

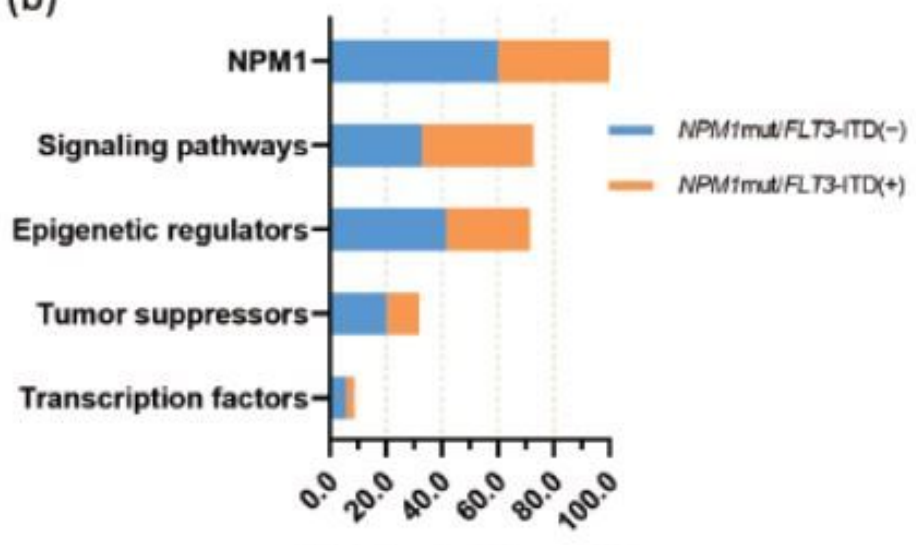

Mutation incidence $(\%)$

Figure 2

Number of mutated genes per patient (a) and incidence of gene functional categories (b) in NPM1 mut AML (by FLT3-ITD). 


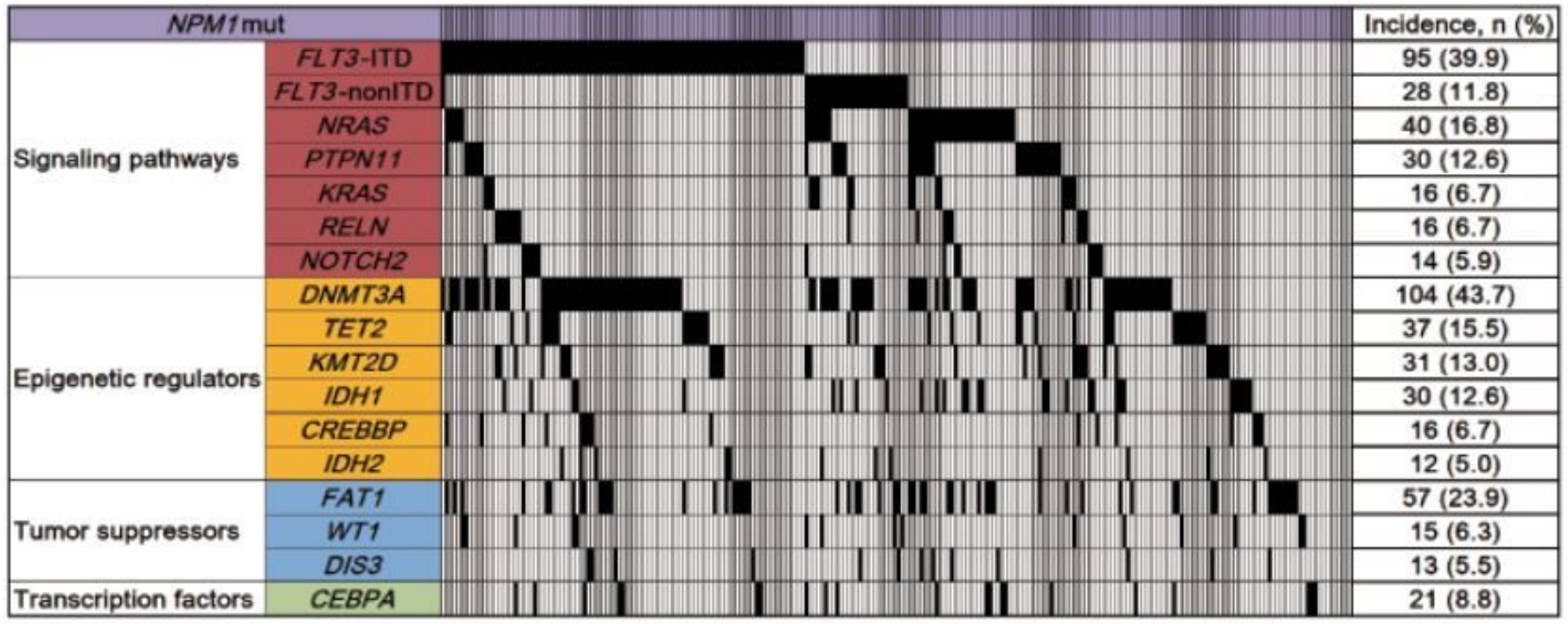

Figure 3

Mutational landscape for the relatively common mutated genes in NPM1mut AML; Row represents each different gene, and column represents each patient subject; Black cell indicates a presence of mutation, and blank cell wild type; The 17 individual genes were grouped into seven functional categories as labeled on the left, and mutational incidence of each gene was listed on the right.

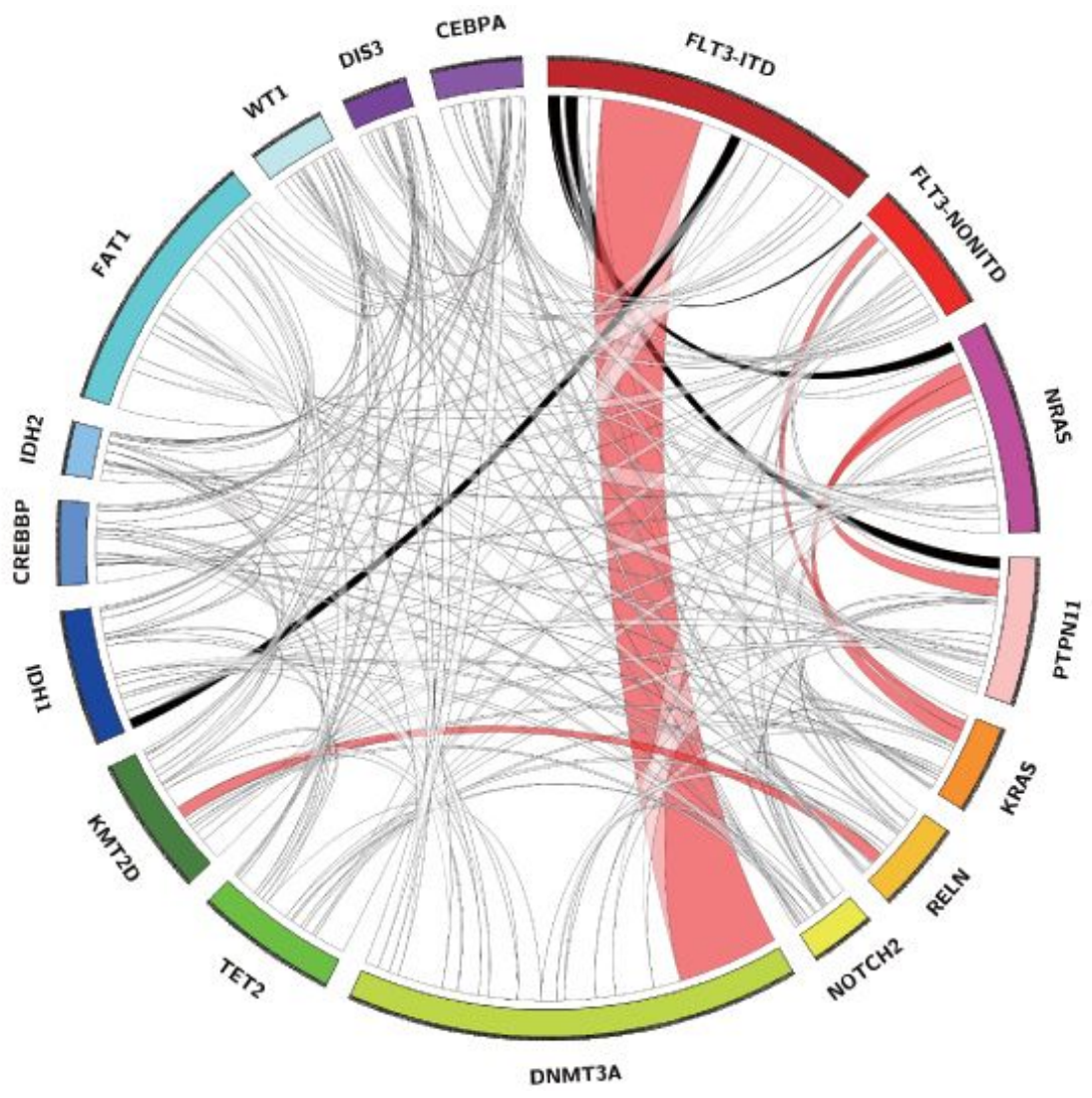

Figure 4 
Circos plot illustrating pairwise relationships across the relatively common mutated genes in NPM1mut AML; Red ribbon indicates a significant coexistence, and black ribbon a mutual exclusivity; White ribbon indicates a non-significant association; Width of ribbon corresponds to number of patients who have simultaneous presence of a first and a second gene in parallel. 